\title{
Electricity Spot Price Dynamics: Beyond Financial Models *
}

\author{
Graeme A. Guthrie ${ }^{\dagger}$ and Steen Videbeck \\ New Zealand Institute for the Study of Competition and Regulation, \\ and Victoria University of Wellington
}

December 14, 2004

${ }^{*}$ The authors gratefully acknowledge the helpful comments of Lewis Evans, Dinesh Kumareswaran, Peter Thomson, and participants of the Advanced Workshop in Regulation and Competition, 23 ${ }^{\text {rd }}$ Annual Eastern Conference, Pennsylvania, USA. M-co New Zealand generously provided the data used in this study. The views expressed in this paper are those of the authors alone.

${ }^{\dagger}$ Corresponding author. Address: School of Economics and Finance, Victoria University of Wellington, PO Box 600, Wellington, New Zealand. Ph: 64-4-4635763. Fax: 64-4-4635566. Email: graeme.guthrie@vuw.ac.nz 


\title{
Electricity Spot Price Dynamics: Beyond Financial Models
}

\begin{abstract}
An increasing number of researchers attempt to model the behavior of electricity spot prices using statistical models commonly used to model financial asset prices. In this paper we reveal properties of electricity spot prices which such models cannot capture. Using six years of half-hourly price data from the New Zealand Electricity Market, we find that the half-hourly trading periods fall naturally into five groups corresponding to the overnight off-peak, the morning peak, daytime off-peak, evening peak, and evening off-peak. The prices in different trading periods within each group are highly correlated with each other, yet the correlations between prices in different groups are lower. Models, adopted from the modelling of security prices, that are currently applied to electricity spot prices are incapable of capturing this behavior. We use a periodic autoregression to model prices instead, showing that shocks in the peak periods are larger and less persistent than those in off-peak periods, and that they often reappear in the following peak period. In contrast, shocks in the offpeak periods are smaller, more persistent, and die out (perhaps temporarily) during the peak periods. Current approaches to modelling spot prices cannot capture this behavior either.
\end{abstract}

JEL Classification code: G13, L94, Q4

Keywords: Electricity markets, spot prices, periodic autoregression 


\section{Electricity Spot Price Dynamics: Beyond Financial Models}

\section{Introduction}

The last ten years have seen a wave of deregulation sweep through the world's electricity markets. As a result, electricity prices in many regions are now determined by the forces of demand and supply. Industry participants need to understand the behavior of deregulated prices in order to price the growing array of electricity derivatives being traded and in order to implement the real option models that are increasingly being advocated for investment and generation decisions in the electricity sector. ${ }^{1}$ Modelling prices has therefore become an active area of research, and the use of statistical models commonly applied to financial time series is popular. ${ }^{2}$ In this paper we argue that the models used to date are not capable of capturing key price behavior, which we document.

The starting point for our analysis is the recognition that its non-storability means that electricity traded at a particular time of the day is a distinct commodity, quite different from electricity traded at different times. This leads us to analyze the behavior of prices in individual trading periods, an approach made possible by the availability of six years of half-hourly price data from the New Zealand Electricity Market. ${ }^{3}$ We find that the half-hourly trading periods fall naturally into five groups corresponding to the overnight off-peak, the morning peak, daytime off-peak, evening peak, and evening off-peak. The prices in different trading periods within each group are highly correlated with each other, yet the correlations between prices in different groups are lower. ${ }^{4}$ These intra-period correlation patterns cannot be captured by standard financial models of spot prices, which assume that the correlation between prices in different trading periods depends only on the length of time between these periods, and not on the time at which the prices are observed. In fact, the presence of these patterns in conventional financial markets would be taken as evidence of market inefficiency, since they would allow investors to make abnormal profits from buying and selling securities at different times of the day. Such strategies are not feasible in electricity markets because electricity is not storable.

The correlation structure identified here is of practical importance for the way generation

\footnotetext{
${ }^{1}$ See, for example, Deng (2000) on derivative pricing and Tseng and Barz (2002) on real option valuation.

${ }^{2}$ Examples of statistical models of electricity spot and forward prices include Bhanot (2000); Burger et al. (2004); Karolyi and Goto (2004); Knittel and Roberts (2004); Lucia and Schwartz (2001). Bessembinder and Lemmon (2002) develop an equilibrium model of electricity forward prices. Longstaff and Wang (2003) test some of their predictions and analyze other properties of forward and spot electricity prices in the PJM market in the mid-Atlantic area of the US.

${ }^{3}$ Some other authors have followed a similar approach when analyzing high frequency electricity price data. For example, Bhanot (2000) models peak and off-peak prices separately. Longstaff and Wang (2003) use a vector autoregression to model the behavior of the 24 individual series of hourly spot prices in their data set.

${ }^{4} \mathrm{Li}$ and Flynn $(2004 \mathrm{a}, 2004 \mathrm{~b})$ identify intraday patterns in average prices for a large number of international electricity markets. Knittel and Roberts (2004) and Lucia and Schwartz (2002) find similar patterns in price data from California and the Nordic Power Exchange respectively.
} 
decisions should be made. For example, if the price is higher than average at midnight, it is likely to be higher than average for the remainder of the overnight period. However, the midnight price reveals little about the price in the morning peak period. For plants which cannot be turned on and off instantaneously, there is an advantage in delaying generation decisions until after the start of one of these intra-day markets. For example, there is likely to be some value in keeping a moderately inflexible plant operating past the end of the morning peak period, since the mid-morning price reveals a great deal of information concerning prices through until the evening peak.

We believe that a new approach is required if these patterns in prices are to be modelled satisfactorily. One possibility, which we illustrate in this paper, is the use of a periodic autoregressive model. We use this approach to show that shocks in the peak periods are larger and less persistent than those in off-peak periods, and that they often reappear in the following peak period. In contrast, shocks in the off-peak periods are smaller, more persistent, and die out (perhaps temporarily) during the peak periods. A simple $\mathrm{AR}(1)$ process, which ignores the different behavior of prices in different trading periods, can be calibrated to capture the low persistence evident in peak periods, or the greater persistence in off-peak periods, but not both simultaneously. Nor can it capture the reappearance of shocks later in the day when they first appear.

\section{Data}

The New Zealand Electricity Market (NZEM) operates a network comprising 244 nodes, each with its own price calculated for each of the 48 daily half hour trading periods. The pricing algorithm used to calculate these nodal prices implements a uniform price auction using actual metered data, final energy and reserve offers, and grid configuration. The price at each node equals the marginal cost of supplying one additional unit of electricity to that node, with differences in nodal prices caused by transmission losses and grid constraints.

This paper uses all 48 final spot prices each day at the Haywards node, located between large scale generation in the south of the South Island and high load areas in the north of the North Island. ${ }^{5,6}$ Our data set comprises every price in the period 1 January 1997 to 12 December 2002, or 105,168 observations in total. From the raw time series data we extract 48 separate series of prices, each one corresponding to a different trading period, with trading period one beginning at midnight, period two beginning at 12:30 a.m., and so on. Each series has one observation a day for each trading period for six years.

There are strong seasonal patterns in the data, so our analysis uses filtered prices, taken as

\footnotetext{
${ }^{5}$ There are three types of prices in the NZEM: forecast prices, which are calculated during the 36 hours before the particular trading period; dispatch prices, which are calculated in the final few hours before electricity is dispatched; and final prices, which are generally available on the following day and are used for settlement. This study uses final prices as they most accurately reflect the prices which industry players face.

${ }^{6}$ Other studies, notably Escribano et al. (2002) and Wolak (1999) also examine the behavior of prices at this node.
} 
Figure 1: Intra-day correlation structure

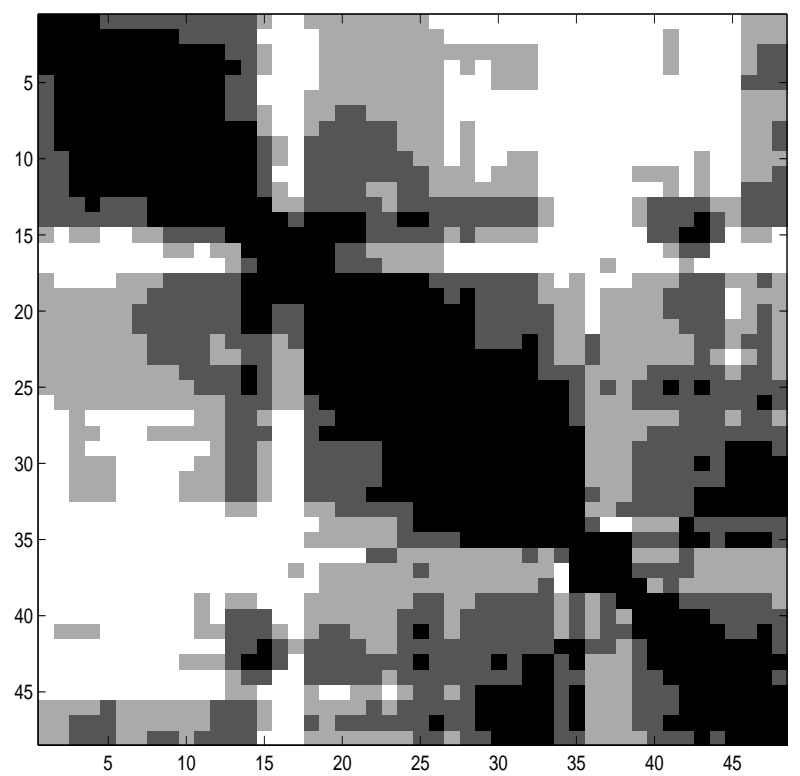

Notes. The graph displays the correlation matrix for filtered prices at the Haywards node over the period 1997-2002. The cells shaded in black correspond to those combinations of trading periods for which the correlation between filtered prices is in the upper quartile of all correlation coefficients, the cells shaded dark gray have correlations in the upper middle quartile, and so on.

the residuals from the regression

$$
p_{n, t}=\sum_{i=1}^{7} \alpha_{n, i} d_{i, t}+\sum_{i=1}^{71} \gamma_{n, i} m_{i, t}+\varepsilon_{n, t}, \quad \varepsilon_{n, t} \sim N\left(0, \psi_{n}^{2}\right),
$$

where $p_{n, t}$ is the price in trading period $n$ on day $t, d_{j, t}$ is a dummy variable that takes the value 1 on day $j$ and 0 otherwise, $m_{j, t}$ is a dummy variable that takes the value 1 in month $j$ and 0 otherwise (January 1996 is month 1, January 1997 is month 13, and so on).

\section{Intra-day correlations}

Our prime focus in this paper is the structure of correlations between prices in different trading periods. $^{7}$ The $48 \times 48$ correlation matrix for our sample period is displayed graphically in Figure 1. The cells in the grids each correspond to an element of the correlation matrix. The shade of the cell indicates the strength of correlation: cells shaded in black correspond to those combinations of trading periods for which the correlation between filtered prices is in the upper quartile of all correlation coefficients, the cells shaded dark gray have correlations in the upper middle quartile, and so on. The graph reveals a remarkably rich structure, with the trading periods falling naturally into groups: the prices in different trading periods within each group

\footnotetext{
${ }^{7}$ Lucia and Schwartz (2002) find that the 24 series of hourly prices on the Nordic Power Exchange are all highly correlated $(\rho>0.94)$, but do not report the existence of pattern to the correlation structure.
} 
Table 1: Average correlation coefficients across the day

\begin{tabular}{lccccc}
\hline Correlation & Overnight & Morning peak & Daytime & Evening peak & Evening \\
\hline Overnight & 0.64 & 0.34 & 0.36 & 0.23 & 0.34 \\
Morning peak & & 0.69 & 0.38 & 0.27 & 0.34 \\
Daytime & & & 0.61 & 0.38 & 0.46 \\
Evening peak & & & & 0.70 & 0.39 \\
Evening & & & & 0.61 \\
\hline
\end{tabular}

Notes. The entries in the table report the average correlation coefficients for filtered prices in different trading periods at the Haywards node over the period 1997-2002. The overnight period contains trading periods 1-14 (midnight until 7:00 a.m.), the morning peak contains trading periods 15-17 (7:00-8:30 a.m.), the daytime period contains trading periods 18-35 (8:30 a.m.-5:30 p.m.), the evening peak contains trading periods 36-38 (5:30-7:00 p.m.), and the evening period contains trading periods 39-48 (7:00 p.m. until midnight).

are highly correlated with each other, yet the correlations between prices in different groups are lower. ${ }^{8}$ These intra-period correlation patterns cannot be captured by standard financial models of spot prices - that is, in models which assume that the behavior of prices is constant over the course of the day. Specifically, models which assume that the correlation between prices in periods $m$ and $n$ depends only on $n-m$, and not $m$ or $n$ individually, would have correlation matrices with the same value down each diagonal. The graph corresponding to Figure 1 would show a series of downward sloping stripes.

There appear to be five distinct groups of trading periods in Figure 1: overnight (periods 1-14), morning peak (periods 15-17), daytime (periods 18-35), evening peak (periods 36-38), and late evening (periods 39-48). As reported in Table 1, the average correlation coefficient between prices in different trading periods during the overnight period is $0.64 .^{9}$ The average is 0.69 within the morning peak period, 0.61 during the daytime period, 0.70 in the evening peak period, and 0.61 in the evening period. In contrast, the average correlation between overnight prices and those in the morning peak period is 0.34 , and with those in the evening peak period is 0.23 . In fact, the daytime and evening periods could almost be combined into a single daytime off-peak period interrupted by a brief evening peak period.

Further evidence on this correlation structure can be found using principal components analysis. When applied to the 48 price series, the first five principal components account for $73 \%$ of the variance over the six year period. When applied to individual years, the percentage explained by the first five principal components varies from a low of $68 \%$ in 1998 to a high of $89 \%$ in $2002 .{ }^{10}$

\footnotetext{
${ }^{8}$ An even clearer picture emerges when we focus on individual years. See the appendix for details.

${ }^{9}$ We calculate the average correlation coefficient for pairs $\left(p_{i, t}, p_{j, t}\right)$ with $i \neq j$ in the same group.

${ }^{10}$ High correlations between the prices of different commodities indicates that the commodities trade in the same economic market (Stigler and Sherwin, 1985). Thus, we speculate that there are perhaps five separate intra-day markets operating in the NZEM.
} 


\section{A periodic regression model}

In this section we use a variant of a standard autoregression, known as a periodic autoregression (PAR), to model our spot price data. PAR models appear similar to AR models except that the AR coefficients take different values in different trading periods. In our case the filtered price in any trading period is assumed to potentially depend linearly on the filtered prices in the preceding 48 trading periods:

$$
p_{n, t+1}^{*}=\sum_{i=1}^{n-1} \beta_{n, i} p_{n-i, t+1}^{*}+\sum_{i=n}^{48} \beta_{n, i} p_{n+48-i, t}^{*}+u_{n, t+1},
$$

where $p_{n, t}^{*}=p_{n, t}-\hat{p}_{n, t}$ is the residual from the regression in (1). We focus on four different specifications of the PAR model:

Model (i) The price in a particular trading period is regressed on the price in the same trading period on the previous day: ${ }^{11}$

$$
p_{n, t+1}^{*}=\beta_{n, 48} p_{n, t}^{*}+u_{n, t+1}
$$

Model (ii) The price is regressed on the price in the previous trading period: ${ }^{12}$

$$
p_{n, t+1}^{*}=\beta_{n, 1} p_{n-1, t+1}^{*}+u_{n, t+1} .
$$

Model (iii) The price is regressed on the previous day's price in the same trading period and the price in the previous trading period:

$$
p_{n, t+1}^{*}=\beta_{n, 48} p_{n, t}^{*}+\beta_{n, 1} p_{n-1, t+1}^{*}+u_{n, t+1} .
$$

This model combines the features of our first two restricted models, and might provide a parsimonious means of capturing the relationship between prices in different trading periods and on different days.

Model (iv) The price is regressed on all 48 prices recorded in the previous 24 hours. This is the unrestricted model (2).

Pargano (1978) discusses PAR models in detail, and proves that each component equation of a PAR model can be estimated separately. Table 2 reports Akaike's information criterion (AIC) for each of the four models described above, for each trading period. The second column reveals that the price in the previous trading period provides more information than the price in the same period on the previous day. However, while the AICs fall when both of these lagged prices are included, comparison with the fourth column reveals that including all 48 lagged

\footnotetext{
${ }^{11}$ This is similar to the approach of Bhanot (2000), who models the behavior of daily peak and off-peak prices using separate $\mathrm{AR}(1)$ processes.

${ }^{12}$ We are not aware of any previous study using this approach. Knittel and Roberts (2004) regress prices on their one-hour lag, but in their case the autoregressive coefficient, $\beta_{n, 1}$ in model (ii), takes the same value for all values of $n$.
} 
Table 2: Performance of various periodic autoregressive models

\begin{tabular}{rccccccccccccccc}
\hline \multicolumn{1}{c}{ AIC } & \multicolumn{1}{c}{ AIC } & \multicolumn{4}{c}{ AIC } \\
$n$ & (i) & (ii) & (iii) & (iv) & \multicolumn{1}{c}{$n$} & \multicolumn{1}{c}{ (i) } & (ii) & (iii) & (iv) & $n$ & (i) & (ii) & (iii) & (iv) \\
\hline 1 & 8.90 & 8.82 & 8.77 & 8.56 & 17 & 10.28 & 9.11 & 9.11 & 8.82 & 33 & 9.50 & 8.06 & 8.06 & 7.97 \\
2 & 8.17 & 7.80 & 7.71 & 7.47 & 18 & 9.52 & 8.97 & 8.93 & 8.46 & 34 & 9.60 & 9.05 & 9.05 & 8.85 \\
3 & 8.12 & 7.31 & 7.30 & 7.15 & 19 & 9.10 & 8.41 & 8.36 & 8.00 & 35 & 9.91 & 9.28 & 9.28 & 9.06 \\
4 & 8.15 & 7.26 & 7.22 & 7.03 & 20 & 9.08 & 7.82 & 7.81 & 7.63 & 36 & 10.57 & 10.11 & 10.11 & 9.95 \\
5 & 8.11 & 6.97 & 6.94 & 6.74 & 21 & 8.97 & 7.35 & 7.34 & 7.13 & 37 & 10.49 & 9.88 & 9.83 & 9.72 \\
6 & 8.14 & 6.87 & 6.86 & 6.64 & 22 & 9.11 & 8.23 & 8.23 & 7.94 & 38 & 10.34 & 9.23 & 9.21 & 9.08 \\
7 & 8.07 & 6.22 & 6.22 & 6.17 & 23 & 9.09 & 7.02 & 7.02 & 6.96 & 39 & 9.94 & 9.67 & 9.64 & 9.37 \\
8 & 7.83 & 6.38 & 6.36 & 6.24 & 24 & 8.92 & 7.98 & 7.96 & 7.62 & 40 & 9.69 & 9.19 & 9.06 & 8.82 \\
9 & 7.76 & 6.19 & 6.19 & 6.12 & 25 & 8.89 & 7.47 & 7.47 & 7.39 & 41 & 9.62 & 8.09 & 8.03 & 7.93 \\
10 & 7.80 & 6.09 & 6.09 & 6.00 & 26 & 8.76 & 7.58 & 7.56 & 7.39 & 42 & 9.54 & 8.83 & 8.79 & 8.31 \\
11 & 8.01 & 6.67 & 6.66 & 6.39 & 27 & 8.91 & 8.09 & 8.08 & 7.89 & 43 & 9.23 & 8.27 & 8.22 & 7.91 \\
12 & 8.12 & 7.09 & 7.08 & 6.93 & 28 & 8.61 & 7.53 & 7.50 & 7.23 & 44 & 9.34 & 8.68 & 8.67 & 8.49 \\
13 & 8.24 & 7.68 & 7.64 & 7.48 & 29 & 9.03 & 7.93 & 7.93 & 7.85 & 45 & 9.43 & 8.25 & 8.26 & 7.79 \\
14 & 8.63 & 8.23 & 8.04 & 7.65 & 30 & 8.98 & 7.61 & 7.61 & 7.48 & 46 & 8.61 & 7.92 & 7.87 & 7.47 \\
15 & 9.39 & 8.69 & 8.66 & 8.35 & 31 & 8.99 & 6.93 & 6.92 & 6.78 & 47 & 8.66 & 7.37 & 7.35 & 7.15 \\
16 & 10.60 & 10.02 & 10.02 & 9.92 & 32 & 8.95 & 7.39 & 7.39 & 7.12 & 48 & 8.51 & 7.37 & 7.36 & 7.07 \\
\hline
\end{tabular}

Notes. The numbers reported in the table are Akaike's information criterion for regressions of special cases of equation (2). In case (i), only the 48 th of the spot price is included; in case (ii) only the first lag is included; in case (iii) both the first and 48th lags are included; in case (iv) all 48 lags are included. All regressions use observations from the Haywards node during the period 1 January 1997 to 31 December 2002.

prices improves the AIC in all cases. This shows that we lose useful information if we ignore the relationships between the prices in different trading periods. We therefore use our estimate of the unrestricted system in (2) to investigate the dynamics of spot prices in more detail.

We examine the effects of price shocks in individual trading periods and see how they propagate throughout the day. The system (2) can be written in the form

$$
\mathbf{\Gamma} \mathbf{p}_{t+1}^{*}=\mathbf{A} \mathbf{p}_{t}^{*}+\mathbf{u}_{t+1}, \quad \mathbf{u}_{t+1} \sim N(\mathbf{0}, \mathbf{D})
$$

where $\mathbf{p}_{t}^{*}=\left(p_{1, t}^{*}, p_{2, t}^{*}, \ldots, p_{48, t}^{*}\right)^{\prime}$,

$$
\boldsymbol{\Gamma}=\left(\begin{array}{ccccc}
1 & 0 & \ldots & 0 & 0 \\
-\beta_{2,1} & 1 & \ldots & 0 & 0 \\
\vdots & & & & \vdots \\
-\beta_{47,46} & -\beta_{47,45} & \ldots & 1 & 0 \\
-\beta_{48,47} & -\beta_{48,46} & \ldots & -\beta_{48,1} & 1
\end{array}\right), \quad \mathbf{A}=\left(\begin{array}{ccccc}
\beta_{1,48} & \beta_{1,47} & \cdots & \beta_{1,2} & \beta_{1,1} \\
0 & \beta_{2,48} & \cdots & \beta_{2,3} & \beta_{2,2} \\
\vdots & & & & \vdots \\
0 & 0 & \ldots & \beta_{47,48} & \beta_{47,47} \\
0 & 0 & \ldots & 0 & \beta_{48,48}
\end{array}\right)
$$

and $\mathbf{D}$ is diagonal. It follows that the vector of forecast errors when forecasting the next day's prices is

$$
\mathbf{p}_{t+1}^{*}-E_{t}\left[\mathbf{p}_{t+1}^{*}\right]=\boldsymbol{\Gamma}^{-1} \mathbf{u}_{t+1}
$$


Figure 2: Impulse response functions
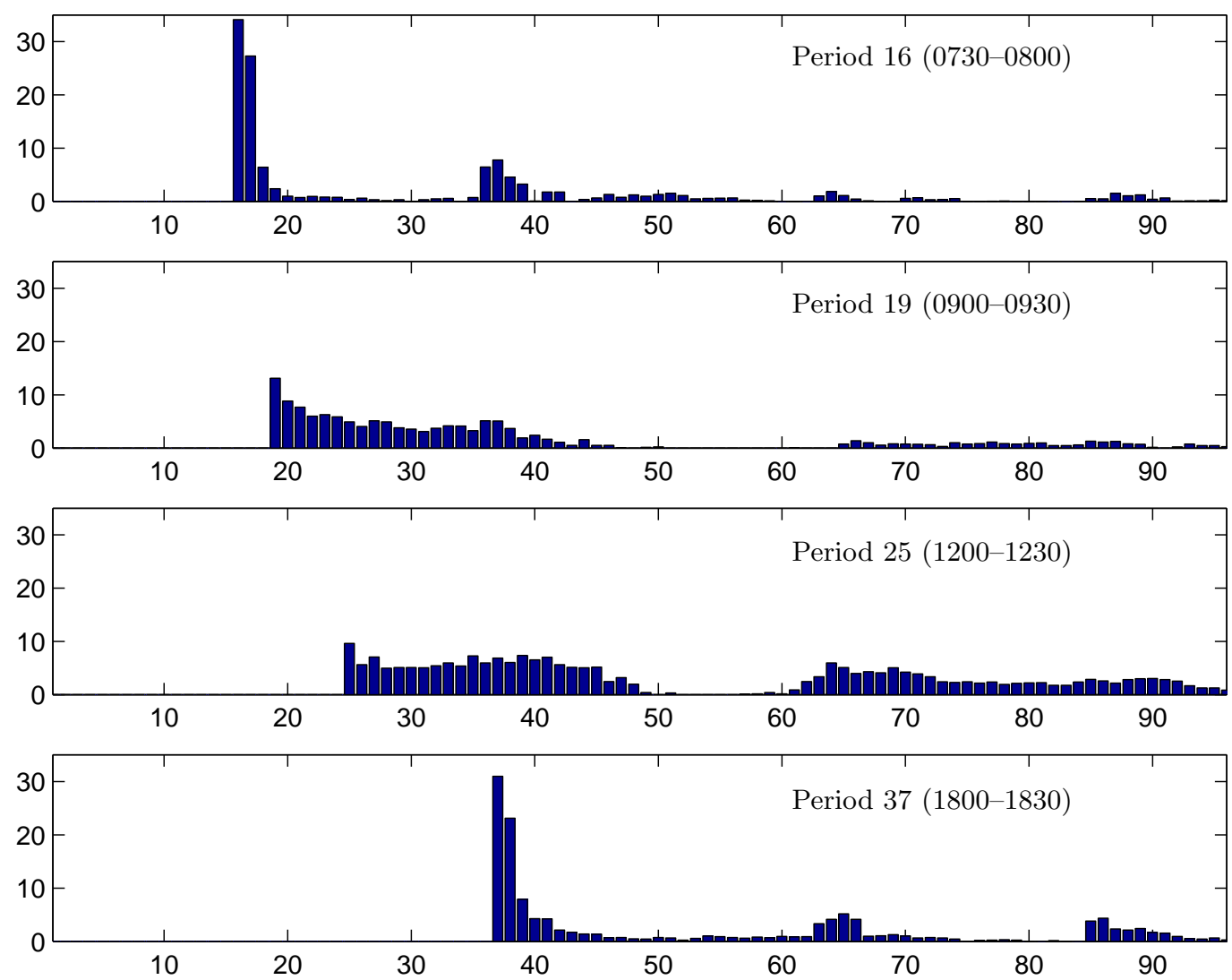

Notes. Each panel shows what happens if the price in the indicated trading period receives a one standard deviation shock. The impulse response functions are derived from our estimates of equation (3) using prices at the Haywards node for the period 1 January 1997 to 31 December 2002.

at the end of day $t$. Similarly, the two-day-ahead forecast error is

$$
\mathbf{p}_{t+2}^{*}-E_{t}\left[\mathbf{p}_{t+2}^{*}\right]=\boldsymbol{\Gamma}^{-1} \mathbf{A} \boldsymbol{\Gamma}^{-1} \mathbf{u}_{t+1}+\boldsymbol{\Gamma}^{-1} \mathbf{u}_{t+2} .
$$

The effect of price shocks for four representative trading periods are plotted in Figure 2. Each graph shows what happens if the spot price in the indicated trading period receives a one standard deviation shock; that is, the graph plots the forecast errors when $\mathbf{u}_{t+1}$ takes the value $\sqrt{d_{n n}}$ in element $n$ and zero elsewhere, where $n$ is the trading period in which the shock occurs and $\sqrt{d_{n n}}$ is the standard error of $u_{n, t+1}$ in (2). From the top panel, shocks during the morning peak period last for two or three trading periods, before disappearing, only to reappear (to some extent) in the subsequent evening peak period. The fourth panel shows that the behavior of price shocks during the evening peak period is similar: lasting two or three trading periods, before quickly disappearing, only to reappear (but without the same strength) in the next day's morning and evening peak periods. In contrast, the second graph shows that shocks occurring immediately after the end of the morning peak period tend to be smaller, but last throughout the day, before permanently disappearing overnight. The third graph suggests that shocks occurring 
in the middle of the day also tend to persist throughout the day and then disappear overnight, although they reappear the following day.

To summarize these findings, it appears that shocks in the peak periods are larger, less persistent, but reappear in the following peak period(s). In contrast, shocks in the off-peak periods are smaller, more persistent, and die out (perhaps temporarily) during the peak periods. This explains the high correlation between prices within off-peak periods, and their low correlation with prices in peak periods, evident in Figure 1. It also provides compelling evidence that the intra-day dynamics are much richer than can be captured by standard models. For example, while a simple $\mathrm{AR}(1)$ process (which ignores the different behavior of prices in different trading periods) can be calibrated to capture the low persistence evident in peak periods, it cannot simultaneously capture the greater persistence in off-peak periods, nor the fact that shocks reappear the following day.

\section{Conclusion}

The unique features of electricity lead us to treat electricity traded at different times as being distinct commodities. We employ a data set comprising six years of half-hourly price data from the New Zealand Electricity Market to analyze the behavior of prices in individual trading periods. We find that the half-hourly trading periods fall naturally into five groups corresponding to the overnight off-peak, the morning peak, daytime off-peak, evening peak, and evening offpeak. The prices in different trading periods within each group are highly correlated with each other, yet the correlations between prices in different groups are lower. We argue that the models currently applied to electricity spot prices are incapable of capturing this behavior.

We illustrate a new approach to modelling electricity prices, the use of periodic autoregressions, in this paper. We use this approach to show that shocks in the peak periods are larger and less persistent than those in off-peak periods, and that they often reappear in the following peak period. In contrast, shocks in the off-peak periods are smaller, more persistent, and die out (perhaps temporarily) during the peak periods. The simple statistical models of spot prices, adopted from the modelling of security prices, cannot capture this behavior.

The periodic autoregression used in this paper could be used to value electricity derivatives with payoffs depending on high frequency spot price dynamics. The PAR's principal limitation, however, is the large number of parameters that need to be estimated. For example, with half-hourly trading periods each of the 48 equations has 48 slope coefficients, in additional to the coefficients of various dummy variables. However, much of the dynamic structure would remain if only a subset of the lagged prices (for example 1, 2, 47 and 48 lags) is used instead. This parsimony might allow us to introduce jumps and other relevant properties into the price process. This is a promising line of inquiry. 


\section{References}

Bessembinder, Hendrik, and Michael L. Lemmon (2002). "Equilibrium pricing and optimal hedging in electricity forward markets," Journal of Finance 57(3), 1347-1382.

Bhanot, Karan (2000). "Behavior of power prices: Implications for the valuation and hedging of financial contracts," Journal of Risk 2(3), 43-62.

Burger, Markus, Bernhard Klar, Alfred Müller, and Gero Schindlmayr (2004). "A spot market model for pricing derivatives in electricity markets," Quantitative Finance 4, 109-122.

Deng, Shi-Jie (2000). "Pricing electricity derivatives under alternative stochastic spot price models," Proceeding of the 33rd Hawaii International Conference on System Sciences.

Escribano, Alvaro, J. Ignacio Peña, and Pablo Villaplana (2002). "Modelling electricity prices: International evidence," Working paper 02-27, Universidad Carlos III de Madrid.

Karolyi, G. Andrew, and Mika Goto (2003). "Understanding electricity price volatility within and across markets," working paper, Ohio State University and Central Research Institute of Electric Power Industry of Japan.

Knittel, Christopher R., and Michael Roberts (2004). "Financial models of deregulated electricity prices: An application to the California market," forthcoming in Energy Economics.

Li, Ying, and Peter C. Flynn (2004a). "Deregulated power prices: Comparison of diurnal patterns," Energy Policy 32, 657-672.

Li, Ying, and Peter C. Flynn (2004b). "Deregulated power prices: Comparison of volatility," Energy Policy 32, 1591-1601.

Longstaff, Francis, and Ashley Wang (2003). "Electricity forward prices: A high frequency empirical analysis," forthcoming in Journal of Finance.

Lucia, Julio J., and Eduardo S. Schwartz (2002). "Electricity prices and power derivatives: Evidence from the Nordic power exchange," Review of Derivatives Research 5, 5-50.

Pargano, M. (1978). "On periodic and multiple regressions," Annals of Statistics 6(6), 13101317.

Stigler, G. J., and R. A. Sherwin (1985). "The extent of the market," Journal of Law and Economics 28, 555-586.

Tseng, Chung-Li, and G. Barz (2002). "Short-term generation asset valuation: A real options approach," Operations Research 50(2), 297-310.

Wolak, Frank A. (1999). "Market design and price behavior in restructured electricity markets: An international comparison," in Ito, Takatoshi, and Anne Krueger (editors) (1999), Competition Policy in the Asia Pacific Region, EASE Volume 8, 79-134. 


\section{Appendix}

Figure 3: Intra-day correlation structure in individual years
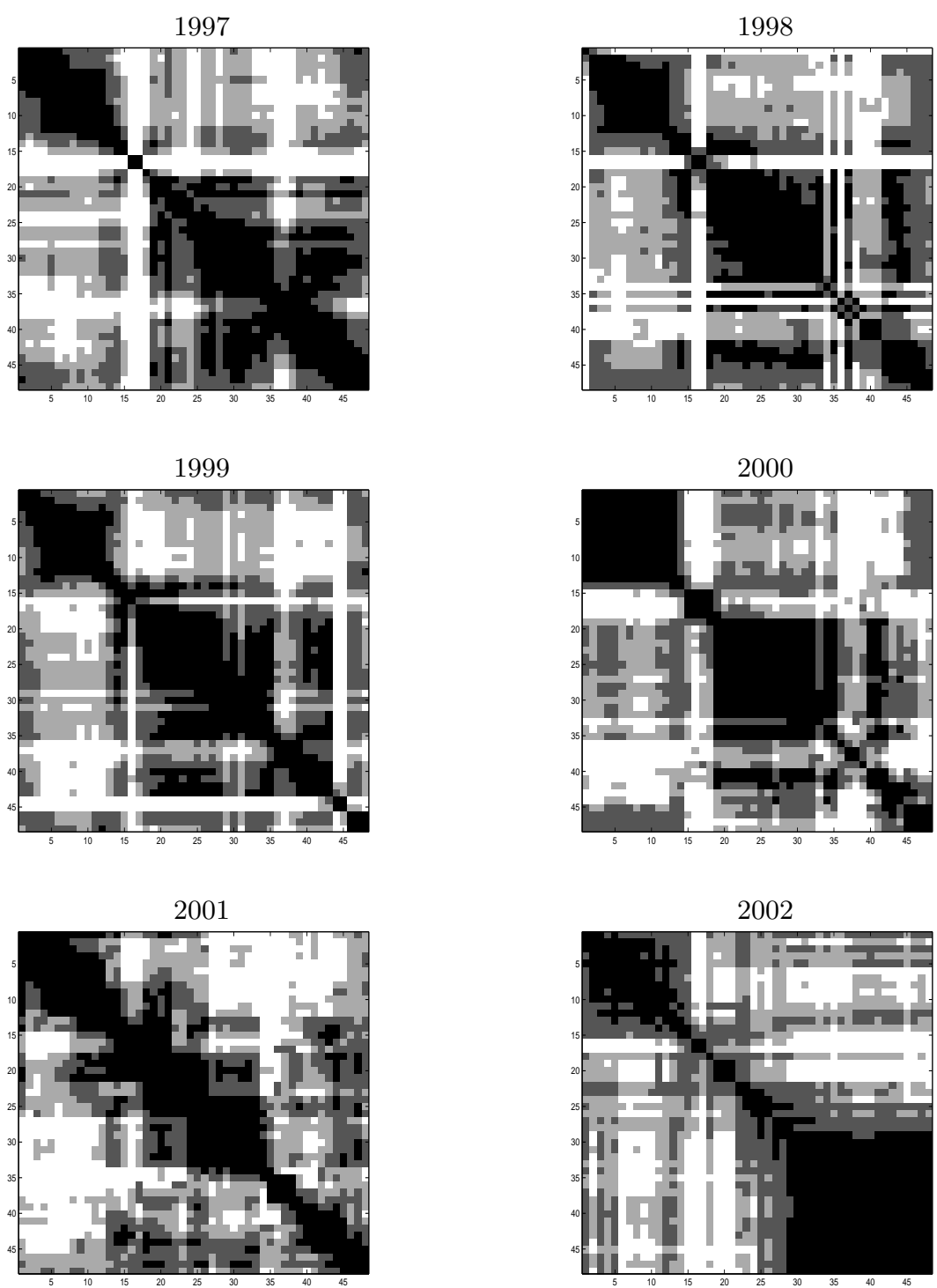

Notes. The graphs display the correlation matrix for filtered prices at the Haywards node for the indicated years. The cells shaded in black correspond to those combinations of trading periods for which the correlation between filtered prices is in the upper quartile of all correlation coefficients, the cells shaded dark gray have correlations in the upper middle quartile, and so on. 